\title{
Relação treinador-atleta e a experiência positiva de jovens no esporte extracurricular
}

\section{Relación entrenador-atleta y experiencia positiva de jóvenes en el deporte extracurricular}

\section{Coach-athlete relationship and youth positive experience in sport extracurricular}

\author{
Tavares, M. A. ${ }^{1,2}$, Fonseca, S. ${ }^{3,4}$, Lopes, A. ${ }^{3,4,5}$, Galatti, L. R. ${ }^{6}$, Reverdito, R. S. ${ }^{1,2}$ \\ ${ }^{1}$ Centro Interdisciplinar de Pesquisas em Esporte e Exercício Físico, Universidade do Estado de Mato \\ Grosso, Cáceres, MT, Brasil; ${ }^{2}$ Programa de Pós-graduação em Educação Física, Universidade Federal \\ de Mato Grosso, Cuiabá, MT, Brasil,; ${ }^{3}$ Universidade Lusófona de Humanidades e Tecnologias, Lisboa, \\ Portugal; ${ }^{4}$ Centro de Investigação em Desporto, Saúde e Desenvolvimento Humano, Vila Real, Portugal \\ ${ }^{5}$ Centro de Investigação em Desporto, Educação Física e Exercício e Saúde, Lisboa, Portugal; \\ ${ }^{6}$ Faculdade de Ciências Aplicadas/Limeira, Programa de Pós-graduação em Educação Física, \\ Universidade Estadual de Campinas, Campinas, SP, Brasil.
}

\section{RESUMO}

A qualidade da construção do ambiente positivo para a prática esportiva estabelecida na relação treinador-atleta é reconhecida para uma experiência positiva dos jovens no esporte, podendo ser determinante para uma prática esportiva prolongada. O objetivo desta pesquisa foi verificar se a qualidade da relação treinador-atleta se relaciona com a percepção de experiência positiva dos jovens no esporte. Participam do estudo 630 atletas (350 masc.; 280 fem.), nas modalidades coletivas de handebol (26\%), voleibol (25\%), futsal (31\%) e basquetebol (18\%), em competição de nível estadual, classificatória para etapa nacional. A idade dos atletas é de $16.06 \pm 0.82$ anos e o tempo médio de prática das respectivas modalidades foi de $4.48 \pm 2.54$ anos. O conjunto de dados foi coletado por meio dos questionários Relacionamento Treinador-Atleta (CART-Q Atleta) e Experiência dos Jovens no Esporte (YES-Y), validados para a língua portuguesa. Apesar da literatura reconhecer a importância da relação treinador-atleta, os resultados encontrados no presente estudo sugerem que a qualidade da relação treinador-atleta não influencia a experiência positiva dos jovens no esporte, no contexto escolar extracurricular.

Palavras chave: Treinador-atleta; Experiência positiva; Jovens atletas; Esporte extracurricular; Pedagogia do Esporte.

\section{RESUMEN}

La calidad de la relación entrenador-atleta es reconocida como una experiencia positiva por jóvenes deportistas, que puede ser decisiva para la manutención en la práctica. El objetivo de esta investigación fue verificar si la calidad de 


\title{
Relação Coach-athlete e experiência positiva da juventude
}

la relación entrenador-atleta está relacionada con la percepción de la experiencia positiva de los jóvenes en el deporte. El estudio se realizó con 630 atletas (350 mas.; $280 \mathrm{fem}$.), en los deportes de equipo: balonmano (26\%), voleibol $(25 \%)$, fútbol sala $(31 \%)$ y baloncesto $(18 \%)$, todos participando en competencia de nivel estatal, clasificatoria para la etapa nacional. La edad de los atletas es de $16.06 \pm 0.82$ años y el tiempo medio de práctica en su deporte fue de $4.48 \pm 2.54$ años. El conjunto de datos se recopiló utilizando los cuestionarios de Relación Entrenador-Atleta (CARTQ Athlete) y Experiencia Juvenil en el Deporte (YES-Y), validados para el idioma portugués. Aunque la literatura reconoce la importancia de la relación entrenador-atleta, los resultados encontrados en el presente estudio sugieren que la calidad de la relación entrenador-atleta no influye en la experiencia positiva de los jóvenes en el deporte, en el contexto escolar extracurricular.

Palabras clave: Entrenador-atleta; Experiencia positiva; Atletas jóvenes; Deporte extracurricular; Pedagogía del Deporte.

\begin{abstract}
The quality of the coach-athlete relationship is recognized as a positive experience by young people in sports, which can be decisive for a long-term sports practice. Our aim was to verify if the quality of the relationship coach-athlete is related to the perception of sport as a positive experience by young athletes. The participants are 630 young athletes (350 male; 280 female) in four different team sports: handball (26\%), volleyball (25\%), futsal (31\%) and basketball (18\%), participating in state competitions, which are qualifying for the national championship. The athletes' age was $16.06 \pm 0.82$ years with an average time of practice of $4.48 \pm 2.54$ years. The data was collected using the CoachAthlete Relationship (CART-Q Athlete) and the Youth Experience in Sport (YES-Y) questionnaires, both validated for the Portuguese language. Although the literature recognizes the coach-athlete relationship as positive, the results found in this study suggest that the quality of the coach-athlete relationship, by itself, does not influences the perception of sports as a positive experience by young people in sports, in the extracurricular school context.
\end{abstract}

Keywords: Coach; Positive experience; Young athletes; Extracurricular sport; Sport Pedagogy.

\section{INTRODUÇÃO}

Os programas extracurriculares esportivos são considerados um contexto propício para o desenvolvimento positivo (Aoyagi et al., 2020; Côté \& Hancock, 2016; Danish, Forneris, Hodge, \& Heke, 2004; Gould \& Carson, 2008; Grubertt et al., 2020; Holt et al., 2017; Vella, Oades, \& Crowe, 2011), constituindo-se em um dos ambientes mais acessíveis para os jovens (Hansen \& Larson, 2007). Contudo, inúmeros fatores podem influenciar a experiência dos jovens no esporte (Reece et al., 2020), como o apoio familiar, acesso e continuidade de programas de prática esportiva, formação dos treinadores, qualidade das relações interpessoais estabelecidas no ambiente de treino (Coutinho, Mesquita, \& Fonseca, 2018; Gaion et al., 2020; Reverdito et al., 2016; Sheridan, Coffee, \& Lavallee, 2014). Nesse estudo, o foco está sobre a qualidade da relação treinador-atleta na experiência positiva dos jovens no esporte.

A participação do treinador na estruturação de ambientes facilitadores para a qualidade das relações interpessoais é apontado como um elemento importante para o engajamento e permanência na prática esportiva (Lima, 2018; MacDonald, Côté, \& Deakin, 2010; Reverdito, Galatti, Strachan, Scaglia, \& Paes, 2020b), mobilizando aspectos importantes do processo de desenvolvimento de atletas (Camiré, Rathwell, Turgeon, \& Kendellen, 2019; Gould \& Carson, 2010; Santos, Camiré, \& Campos, 2018). A valorização e promoção de treinos pautados no desenvolvimento positivo dos jovens (Holt et al., 2017; Santos et al., 2018), tem sido reconhecido como facilitador para a transferência de habilidades ao longo da vida. No ambiente da prática esportiva os treinadores têm o poder de influenciar a natureza e a qualidade das experiências esportivas, o que reforça a missão de educar, estimular e criar oportunidade para o crescimento e desenvolvimento pessoal dos atletas (Côté, Strachan, \& Fraser-Thomas, 2008; Naylor, 2006; Santos, Gould, \& Strachan, 2019).

A qualidade da relação treinador-atleta tem sido associada a experiências positivas de prática esportiva em diferentes contextos (Jowett \& Cockerill, 2003; Mollerlokken, Loras, \& Pedersen, 2017; Yang \& Jowett, 2012). A coesão de grupo (Jowett \& Chaundy, 2004; Rodrigues, Evans, \& Galatti, 2019), 


\section{Tavares, M. A.; Fonseca, S.; Lopes, A.; Galatti, L. R.; Reverdito, R. S.}

melhorias nas habilidades físicas e psicossociais (Jowett, 2005), superação de momentos críticos (Jowett, 2009), eficácia coletiva (Hampson \& Jowett, 2014), qualidade do treino (Reverdito et al., 2020b), satisfação dos atletas, estratégias de comunicação (Davis, Jowett, \& Tafvelin, 2019) e inteligência emocional (Benito, Francisco, \& Trigueros, 2018), são algumas das temáticas associadas à qualidade das relações interpessoais entre o treinador e os/as atletas. Da mesma forma, estudos apontam efeitos negativos da experiência da participação dos jovens no esporte associados ao treinador (Orozco, Perez, Sierra, Supervia, \& Jarie, 2018; Reverdito, Galatti, Scaglia, \& Paes, 2020a; Wachsmuth, Jowett, \& Harwood, 2017), tendo o abandono precoce da prática esportiva como uma das principais consequências. Entretanto, é possível reconhecer na literatura consenso sobre a importância da qualidade da relação treinador-atleta para uma experiência positiva dos jovens no esporte e o desenvolvimento de habilidades para a vida.

A relação treinador-atleta refere-se a uma situação em que os aspectos afetivos, cognitivos e comportamentais dos treinadores e atletas se associam de maneira interdependente (Jowett \& Poczwardowski, 2007). Yang e Jowett (2012) caracterizam a relação treinador-atleta ao nível da proximidade, comprometimento e complementaridade. Essas dimensões, interligadas, definem a qualidade da relação treinador-atleta e descrevem o constructo psicológico de caráter interpessoal da relação. A proximidade descreve o sentido afetivo e emocional, com referência ao respeito, confiança e admiração. $\mathrm{O}$ comprometimento mensura a intenção do treinador e atleta de manterem o relacionamento por muito tempo. A complementaridade refere-se aos comportamentos de cooperação, ligação, aceitação e responsividade que os atletas adotam e são desencadeados pelo treinador (Vieira et al., 2015b). Quanto mais os treinadores e atletas se manterem comprometidos, desenvolverem uma amizade positiva, cooperativa e equilibrada, maior a interdependência entre ambos (Mata \& Gomes, 2016), consequentemente, espera-se melhor desenvolvimento positivo dos jovens no esporte.

A participação no esporte tem sido associada positivamente ao desenvolvimento da iniciativa (Larson, 2000), habilidades de liderança (Wright \& Cote, 2003), satisfação com a vida (Gilman, 2001), estabelecimentos de metas (Papacharisis, Goudas,
Danish, \& Theodorakis, 2005), construção do caráter (Light \& Light, 2006; Camiré \& Trudel, 2010), desenvolvimento da identidade (Coatsworth \& Conroy, 2009), habilidades cognitivas, pessoais e sociais (Hansen, Larson, \& Dworkin, 2003) e o relacionamento entre pares (Smith, Ullrich-French, Walker, \& Hurley, 2006). Ao revisar estudos que analisaram as atividades extracurriculares estruturadas, Larson (2000) afirma que o esporte é um contexto fértil para o desenvolvimento positivo, uma vez que possibilita aos jovens a vivência da motivação intrínseca e a concentração, associando essas atitudes ao desenvolvimento da iniciativa.

O contexto esportivo é um cenário para o engajamento dos jovens em uma atividade significativa e propício para promover o bem-estar (Gould \& Carson, 2008; Santos \& Martinek, 2018; Turnnidge, Côté, \& Hancock, 2014). Contudo, para que as experiências sejam facilitadoras do desenvolvimento, faz-se necessário que o responsável por essa prática ofereça aos atletas o acesso ao esporte de forma organizada e sistematizada (Larson, 2000), uma vez que a estrutura e o contexto da atividade são um dos fatores que determinam a qualidade da participação (Galatti et al., 2017). É preciso que seja realizado de forma intencional pelo treinador, oferecendo diferentes níveis de intensidade no envolvimento e continuidade da prática esportiva.

As experiências positivas são influenciadas pela intensidade e o tempo de prática. Reverdito et al. (2017a) afirmam que quanto maior o período dos jovens participando de uma prática esportiva, maiores serão os benefícios desencadeados em relação ao conhecimento e o domínio das habilidades (Larson, Hansen, \& Moneta, 2006). Reverdito et al. (2020b), por sua vez, indicam que também a permanência por período prolongado do treinador favorece $\mathrm{o}$ desenvolvimento positivo de jovens. Da mesma forma, quanto mais tempo os jovens se mantêm engajados em uma atividade, maior será o comprometimento e o envolvimento nas atividades, contribuindo para a qualidade das experiências adquiridas (Holt, 2008; Holt et al., 2017). O pressuposto é que um ambiente harmônico e de relações interpessoais positivas (Mageau \& Vallerand, 2003), intencionalmente estruturado, seja mobilizador de experiências positivas dos jovens no esporte (MacDonald, Cote, Eys, \& Deakin, 2012). As experiências positivas são compreendidas como 


\section{Relação Coach-athlete e experiência positiva da juventude}

situações de desenvolvimento capazes de promover o bem-estar (Larson, Perry, Hang, \& Walker, 2011; Larson \& Tran, 2014), e são preditoras da coesão social e de tarefas entre os jovens (Nascimento Junior et al., 2019).

Apesar dos estudos considerarem a qualidade da relação treinador-atleta como decisiva para o desenvolvimento de experiências positivas dos jovens no esporte (Gaion et al., 2020; Hampson \& Jowett, 2014; Jowett \& Cockerill, 2003; Nicholls, Earle, Earle, \& Madigan, 2017; Yang \& Jowett, 2012), não encontramos estudos que tenham observado de forma sistemática a relação treinador-atleta e a experiência dos jovens no contexto do esporte escolar brasileiro. Assim, o objetivo é verificar se a qualidade da relação treinador-atleta se relaciona com a percepção de experiência positiva dos jovens no esporte. A compreensão desta relação poderá favorecer a estruturação de ambientes de desenvolvimento capazes de promover o bem-estar dos jovens em contexto de prática esportiva.

\section{MATERIAIS E MÉTODOS}

\section{Participantes}

Participaram do estudo 630 atletas, do gênero masculino $(\mathrm{n}=350,56 \%)$ e feminino $(\mathrm{n}=280,44 \%)$, na fase estadual dos Jogos Escolares da Juventude (JEJ) do Estado de Mato Grosso/Brasil, nas modalidades coletivas de handebol (26\%), voleibol (25\%), futsal (31\%) e basquetebol (18\%), com média de idades de $16.06 \pm 0.82$ anos. O tempo médio de prática da modalidade pelos atletas foi de $4.48 \pm 2.54$ anos. Os JEJ são uma ação do Comitê Olímpico Brasileiro para promoção do esporte no contexto escolar, desenvolvido em 4 fases: fase municipal - evento entre as escolas no município; fase regional - escolas campeãs na fase municipal; fase estadual - escolas campeãs da fase regional; fase nacional - escolas campeãs da fase estadual.

\section{Instrumentos}

Foram utilizados os questionários Relacionamento Treinador-Atleta (CART-Q Atleta) (Vieira et al., 2015b) e Experiência Positiva dos Jovens no Esporte (YES-Y) (Rigoni, 2014), ambos adaptados e validados para a língua portuguesa. $\mathrm{O}$ questionário CART-Q Atleta (Vieira et al., 2015b), em Escala Likert, tem 11 itens que representam as dimensões de proximidade $(\alpha=.83)$, comprometimento $(\alpha=.64)$ e complementaridade $(\alpha=.80) . \quad \mathrm{O}$ questionário YES-Y utilizado foi adaptado para a língua portuguesa e validado por Rigoni (2014), a partir da versão de MacDonald et al. (2012), sendo composto por 18 itens que representam as dimensões de habilidades pessoais e sociais $(\alpha=.57)$, habilidades cognitivas $(\alpha=.67)$, estabelecimento de metas $(\alpha=.68)$ e iniciativa $(\alpha=.61)$, e a dimensão maior, YES-Y Geral $(\alpha=.82)$. A consistência interna dos questionários apresentou indicador de confiabilidade aceitável.

Os instrumentos acima descritos já foram aplicados em diferentes culturas e ambientes de prática esportiva, apresentando boa confiabilidade (Cronin \& Allen, 2015; Gould, Flett, \& Lauer, 2012; Reverdito et al., 2017a; Rigoni, 2014; Vieira et al., 2015b). Assumimos assim que os instrumentos em causa captam a percepção dos efeitos da experiência da pessoa no contexto da prática esportiva.

\section{Procedimentos para coleta dos dados}

Foi realizado contato com a organização do evento e treinadores para apresentar os objetivos da investigação e procedimentos para coleta dos dados. Os questionários foram aplicados individualmente aos atletas, em ambiente reservado e silencioso, entre o primeiro e segundo jogo do evento esportivo. Os jovens foram orientados sobre como responder, bem como a prestar o máximo de atenção e serem sinceros ao registrar suas percepções. Durante o preenchimento dos questionários não houve intervenção. Para padronizar o processo e garantir padrões éticos e científicos, a equipe de pesquisa foi treinada em procedimentos de coleta de dados anteriormente à realização desta coleta.

Em relação à livre e espontânea participação nos estudos, os atletas e seus respectivos responsáveis manifestaram consentimento livre e esclarecido. O estudo foi aprovado em Comitê de Ética em Pesquisa envolvendo Seres Humanos da Universidade do Estado de Mato Grosso (CAAE: 55729716.7.0000.5166).

\section{Análises dos dados}

A análise descritiva é apresentada para todas as variáveis de interesse (Tabela 1), sendo apresentadas a média e o desvio padrão ( $\mathrm{M} \pm \mathrm{DP})$, bem como teste de normalidade. Relativamente à análise inferencial, foram aplicados testes de correlação paramétrica ( $\mathrm{R}$ de Person) e não paramétrica ( $\mathrm{R}$ de Spearman) para a análise da relação entre variáveis 


\section{Tavares, M. A.; Fonseca, S.; Lopes, A.; Galatti, L. R.; Reverdito, R. S.}

quantitativas e testes de comparação de médias entre grupos, nomeadamente o teste t e a ANOVA. O nível de significância foi fixado em $5 \%$.

Tabela 1. Resultado da análise descritiva das dimensões avaliadas na amostra pelos questionários CART-Q e YES-Y

\begin{tabular}{|c|c|c|c|c|c|c|c|}
\hline & Média & $\begin{array}{l}\text { Desvio } \\
\text { Padrão }\end{array}$ & Assimetria & Kurtosis & $\begin{array}{r}\text { Shapiro- } \\
\text { Wilk }\end{array}$ & $\begin{array}{r}P \text {-value of } \\
\text { Shapiro- } \\
\text { Wilk }\end{array}$ & $\begin{array}{r}\text { Alfa de } \\
\text { Cronbach }\end{array}$ \\
\hline \multicolumn{8}{|l|}{$\begin{array}{l}\text { Relação Treinador-Atleta } \\
\text { (CART-Q) }\end{array}$} \\
\hline Proximidade & 6.255 & 0.967 & $-1,705$ & 3.130 & 0.776 & $<.001$ & 0.831 \\
\hline Comprometimento & 5.199 & 1.320 &,- 548 & -0.445 & 0.948 & $<.001$ & 0.645 \\
\hline Complementaridade & 5.879 & 1.067 & $-1,157$ & 1.251 & 0.886 & $<.001$ & 0.809 \\
\hline \multicolumn{8}{|l|}{$\begin{array}{l}\text { Experiência Positiva dos } \\
\text { Jovens no Esporte (YES-Y) }\end{array}$} \\
\hline Habilidades pessoais e sociais & 3.335 & 0.447 &,- 876 & 0.871 & 0.939 & $<.001$ & 0.572 \\
\hline Habilidades cognitivas & 3.154 & 0.575 &,- 610 & 0.139 & 0.955 & $<.001$ & 0.672 \\
\hline Estabelecimento de metas & 3.513 & 0.485 & $-1,220$ & 1.340 & 0.859 & $<.001$ & 0.682 \\
\hline Iniciativa & 3.759 & 0.384 & $-1,862$ & 3.567 & 0.679 & $<.001$ & 0.614 \\
\hline YES-S Geral & 3.395 & 0.373 &,- 968 & 1.342 & 0.944 & $<.001$ & 0.829 \\
\hline
\end{tabular}

\section{RESULTADOS}

De acordo com os resultados na Tabela 2, podemos verificar que o coeficiente de correlação entre as dimensões do questionário CART-Q é forte $(\mathrm{R}>0.60)$ e positivo entre todos os pares de variáveis, indicando que efetivamente existe uma relação linear positiva entre estas dimensões e, portanto, quanto maior for o comprometimento na relação treinadoratleta, maior será a proximidade e a complementaridade. Já os resultados apresentados na Tabela 3, mostram que para as dimensões do questionário YES-Y, não existe uma correlação forte entre elas, embora seja significativa.

Ao considerar os dois prismas (CART-Q e YES-Y Geral) na Tabela 4, verificamos que o grau de correlação entre o índice geral de experiência dos jovens no esporte e as dimensões do CART-Q é fraco $(\mathrm{R}<0.40)$, o que sugere que no contexto investigado, a qualidade da relação treinador-atleta não se relaciona com a percepção do esporte como uma experiência positiva no contexto extracurricular em termos de desenvolvimento de habilidades e comportamentos. 


\section{Relação Coach-athlete e experiência positiva da juventude}

Tabela 2. Resultado da análise de correlação de Pearson entre as dimensões da CART-Q (Relação Treinador-Atleta)

\begin{tabular}{lcc}
\hline & Proximidade & Comprometimento \\
\cline { 2 - 3 } Comprometimento & $0.686^{* * *}$ & $0.683^{* * *}$
\end{tabular}

Nota: $* * * \mathrm{p}<0.001$

Tabela 3. Resultado da análise de correlação de Pearson entre as dimensões da YES-Y (Experiência dos Jovens do Esporte) Habilidades cognitivas Estabelecimento de metas Iniciativa

\begin{tabular}{lcc} 
Habilidades pessoais e sociais & $0.432 * * *$ & $0.510 * * *$ \\
\cline { 2 - 3 } Estabelecimento de metas & $0.493 * * *$ & $0.456 * * * * *$ \\
Iniciativa & $0.403 * * *$
\end{tabular}

Nota: $* * * \mathrm{p}<0.001$

Tabela 4. Resultado da análise de correlação de Pearson entre as dimensões da Relação Treinador-Atleta (CART-Q) e o índice Geral de Experiências dos Jovens no Esporte (YES-Y Geral).

Relação Treinador-Atleta $\quad$ Experiência Jovens no Esporte (YES-Y Geral)

Proximidade

Comprometimento

Complementaridade

Nota: $* * * \mathrm{p}<0.001$.

Na análise comparativa entre gêneros (Figura 1) e (Figura 2), sobre as dimensões dos dois questionários, foi observada diferença significativa $(\mathrm{p}<0.05)$ somente em Iniciativa $(\mathrm{p}=0.03)$. Este resultado sugere que, na generalidade, o fator gênero não influencia a qualidade da relação treinador-atleta (CART-Q), nem a percepção da experiência positiva dos jovens no esporte escolar extracurricular (YES$\mathrm{Y})$.

Em relação à modalidade (Figura 3), foram encontradas diferenças significativas entre a modalidade de handebol e voleibol (Proximidade: $\mathrm{p}<0.001, \quad$ Comprometimento: $\quad \mathrm{p}=0.001$, Complementaridade: $\mathrm{p}<0.001)$, sendo a qualidade da
$0.191 * * *$

$0.287 * * *$

$0.280 * * *$

relação treinador-atleta significativamente melhor na modalidade de handebol em comparação com voleibol. Para a experiência positiva (Figura 4), só foram encontradas diferenças significativas na variável habilidades cognitivas entre o handebol e o futsal $(\mathrm{p}=0.007)$.

Relativamente à forma como os atletas das diferentes modalidades percebem a sua experiência no esporte (Figura 4), verificamos uma relação fraca entre o desenvolvimento das suas habilidades e comportamentos à prática esportiva. Nesse caso, poderá ser revelador que a qualidade da relação treinador-atleta não se relaciona com a percepção da experiência positiva dos jovens no esporte escolar extracurricular investigado. 


\section{Tavares, M. A.; Fonseca, S.; Lopes, A.; Galatti, L. R.; Reverdito, R. S.}

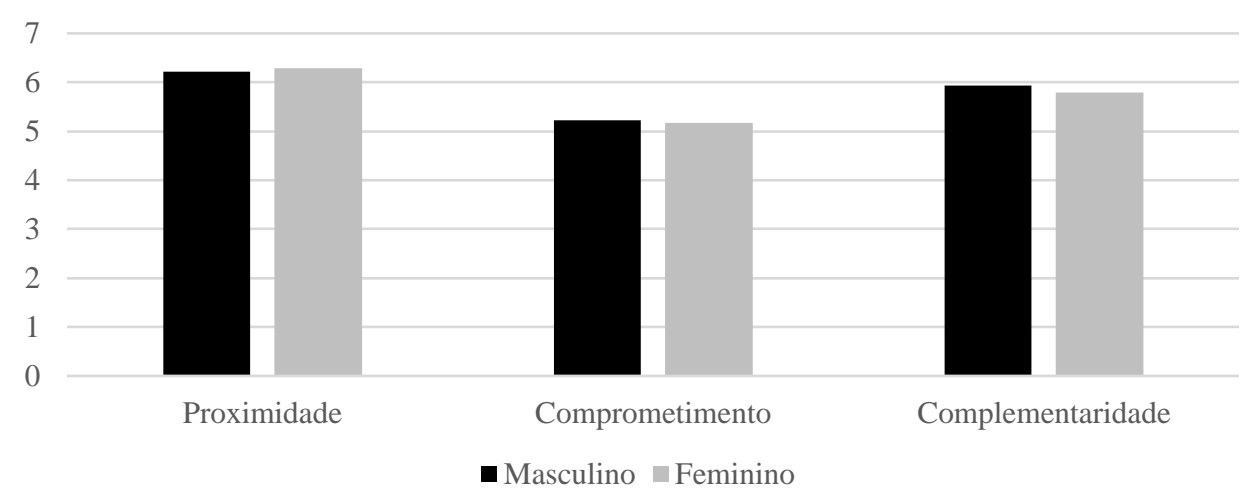

Figura 1. Comparação das dimensões do questionário CART-Q entre gêneros.

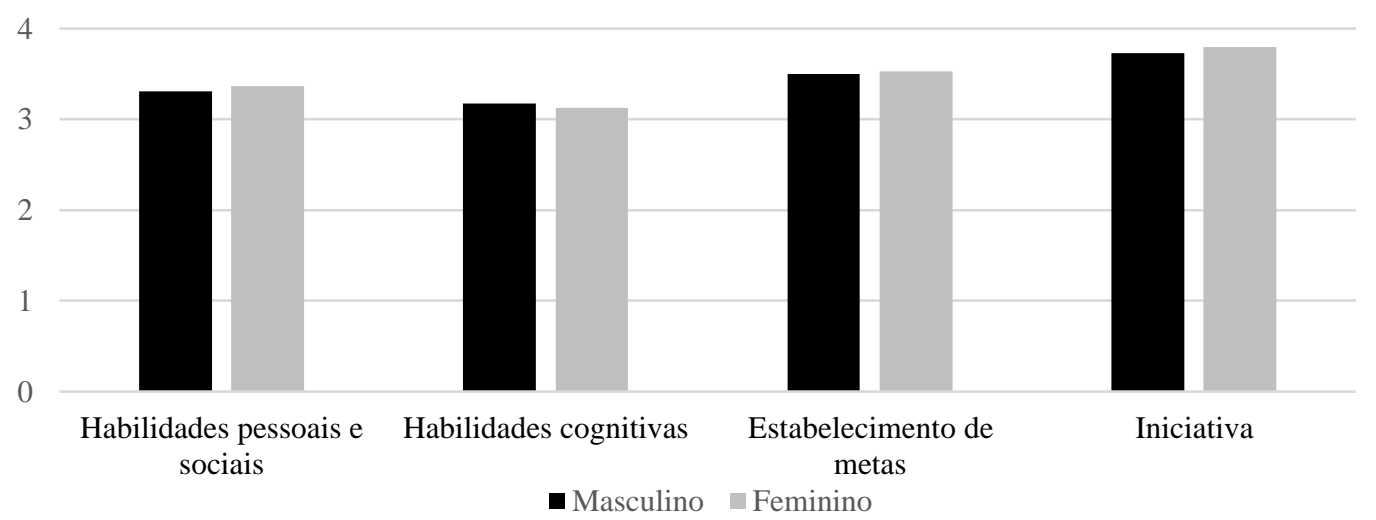

Figura 2. Comparação das dimensões do questionário YES-Y entre gêneros.

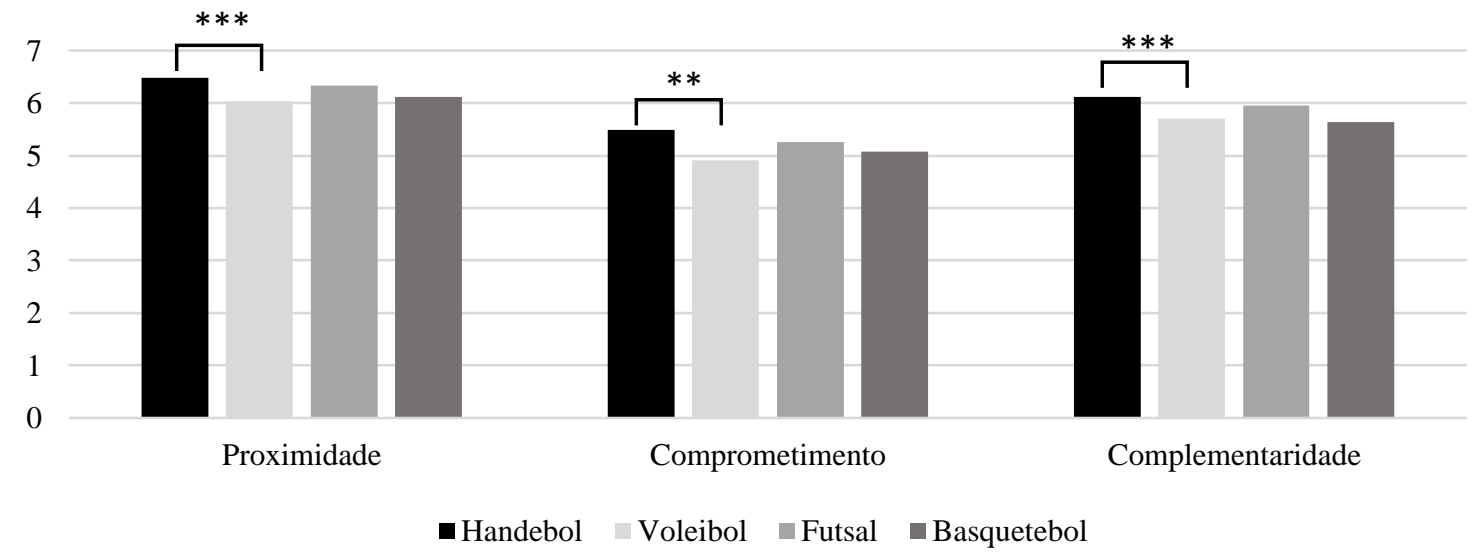

Figura 3. Comparação das dimensões da Relação Treinador Atleta entre modalidades. Os asteriscos indicam as diferenças significativas encontradas (ANOVA) $* * * \mathrm{p}<0.001, * * \mathrm{p}<0.01$. 


\section{Relação Coach-athlete e experiência positiva da juventude}

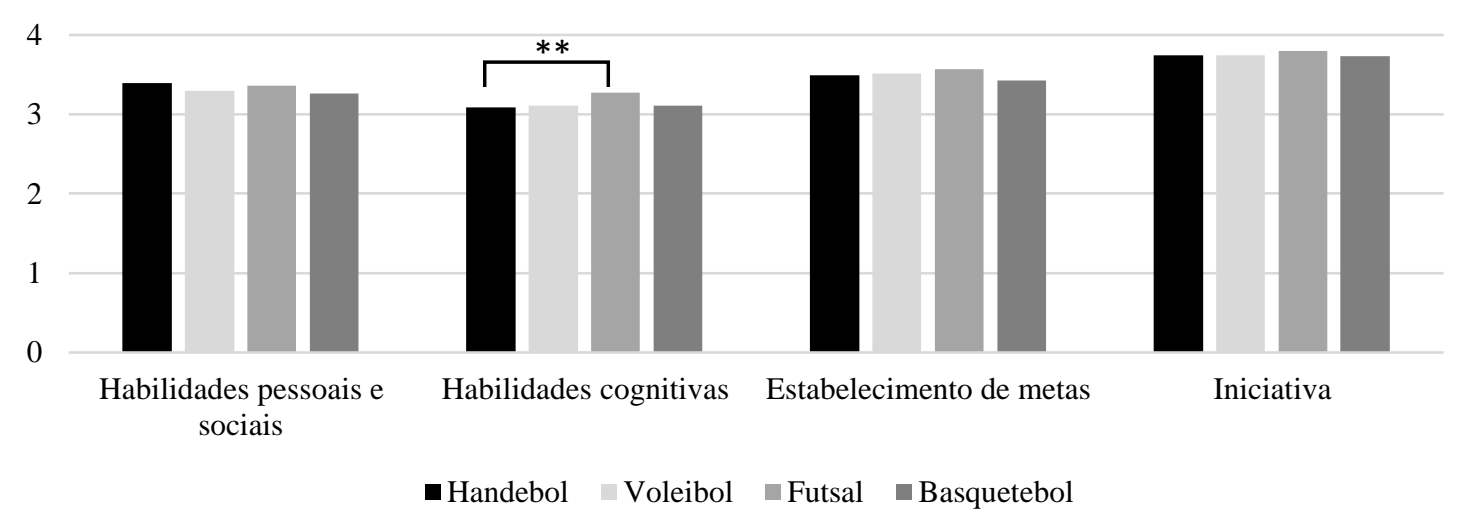

Figura 4. Comparação das dimensões da YES-Y entre modalidades. Os asteriscos indicam as diferenças significativas encontradas (ANOVA) ** $\mathrm{p}<0.01$.

Foi analisada a influência que o tempo de prática do atleta exerce no desenvolvimento das suas habilidades e comportamentos (dimensões do questionário YES-Y) e na qualidade da relação Treinador-Atleta (dimensões do questionário CARTQ Atleta). Através de uma análise de correlação bivariada não paramétrica verificamos que, à exceção da dimensão Estabelecimento de Metas, todas as variáveis avaliadas (Proximidade, Complementaridade, Comprometimento, Habilidades
Pessoais e Sociais, Habilidades Cognitivas, Iniciativa e Índice Geral EJE) apresentam correlações positivas e significativas com o tempo de prática $(p<0.05)$, indicando que quanto mais experiências esportivas positivas, melhores as competências/habilidades e a relação Treinador-Atleta construída. Contudo, e de acordo com os resultados apresentados nas Tabelas 4 e 5, verificamos que as correlações observadas são fracas ( $\mathrm{R}$ de Spearman <0.20) e, portanto, a relação efetiva entre as variáveis não pôde ser inferida.

Tabela 5. Resultado da análise de correlação de Spearman entre o Tempo de Prática (dias) e as dimensões da CART-Q

$$
\text { Proximidade Comprometimento Complementaridade }
$$

\begin{tabular}{llll}
\cline { 2 - 3 } Tempo de prática & $0.097^{*}$ & $0.17 * * *$ & $0.096^{*}$
\end{tabular}

Nota: $* * * \mathrm{p}<0.001, * \mathrm{p}<0.05$.

Tabela 6. Resultado da análise de correlação não paramétrica entre o Tempo de Prática (dias) e as dimensões da YES-Y

$\begin{array}{cccc}\text { Habilidades pessoais e sociais } & \begin{array}{c}\text { Habilidades } \\ \text { cognitivas }\end{array} & \begin{array}{c}\text { Estabelecimento de } \\ \text { metas }\end{array} & \text { Iniciativa }\end{array}$ EJE Geral

\begin{tabular}{lllll}
\cline { 2 - 4 } Tempo de prática & $0.144 * * *$ & $0.1 *$ & 0.051 & $0.082^{*}$
\end{tabular}

Nota: $* * * \mathrm{p}<0.001, * * \mathrm{p}<0.01, * \mathrm{p}<0.05$

\section{DISCUSSÃO}

A qualidade da relação treinador-atleta é reconhecida como fundamental para a participação dos jovens no esporte. Conhecer a influência da relação treinador-atleta na percepção da experiência positiva dos jovens no esporte pode ser determinante para garantir uma prática esportiva prolongada. Nesse estudo, apesar do reconhecimento da importância da relação treinador-atleta (Camiré et al., 2019; Santos et al., 2019; Vella, Oades, \& Crowe, 2013), os resultados encontrados sugerem que a qualidade da relação treinador-atleta não influencia a experiência positiva dos jovens no esporte. Esse resultado difere, em partes, 


\section{Tavares, M. A.; Fonseca, S.; Lopes, A.; Galatti, L. R.; Reverdito, R. S.}

das conclusões dos estudos de Vella et al. (2013), Vieira et al. (2015a) e Fiorese et al. (2018).

Ao identificarem correlação positiva entre a relação treinador-atleta e a experiência positiva dos jovens no esporte, Vella et al. (2013) concluíram que a qualidade da relação treinador-atleta influencia as experiências positivas dos jovens. Vieira et al. (2015a) identificaram que a qualidade da relação treinadoratleta é uma variável importante para a percepção da eficácia coletiva, destacando que os atletas com melhor desempenho competitivo tiveram maior percepção de eficácia coletiva e melhor relação treinador-atleta. Fiorese et al. (2018) verificaram que a relação treinador-atleta é determinante na associação entre a motivação (autônoma e controlada) e a coesão de grupo, constatando que essa relação tem influência sobre diversas variantes.

A explicação para a divergência entre os resultados encontrados e os apresentados por Vella et al. (2013), Vieira et al. (2015a) e Fiorese et al. (2018) pode estar relacionada ao nível de envolvimento na prática esportiva, tempo de prática, regularidade de competição, maior tempo de convívio e idade dos atletas. Os estudos acima citados foram desenvolvidos com participantes fora do contexto escolar, como em clubes. Já os participantes do nosso estudo, ainda que estejam em uma competição de nível estadual, têm sua prática esportiva acontecendo predominantemente no contexto escolar extracurricular. Em comparação com o clube, por vezes, a escola oferece uma prática menos estruturada e menor oportunidade de participação em competições. Em uma prática esportiva mais estruturada e organizada, a relação treinador-atleta poderá exercer maior influência sobre a percepção da experiência positiva dos jovens no esporte.

Ainda, o estudo de Nicholls e Perry (2016) sobre a relação treinador-atleta aponta que a qualidade do relacionamento parece ser mais importante para os treinadores do que para os atletas. Em outro estudo, com jovens em uma liga de futebol, observando mudanças na percepção da relação treinador-atleta e objetivos de realização esportiva ao longo de seis meses, Nicholls et al. (2017) apontam que a relação treinador-atleta foi associada positivamente apenas para o alcance de metas de domínio, por exemplo, na percepção que foi capaz de realizar uma habilidade. É consenso que há benefícios mútuos na qualidade da relação treinador-atleta, mas que precisam ser investigados considerando outros domínios da experiência esportiva, como fatores estressores, nível de competição, tempo de engajamento, motivação para a prática esportiva.

Nossos resultados corroboram com o estudo de Vella et al. (2013) e Vieira et al. (2015a) em relação ao tempo de prática esportiva influenciando positivamente a qualidade da relação treinador-atleta e a experiência positiva dos jovens no esporte. Nesse sentido, os estudos de Reverdito et al. (2017a) e Reverdito et al. (2017b), com jovens no esporte de participação, demonstraram relação entre os efeitos positivos do esporte e o tempo de engajamento. Os jovens com mais de dois anos de prática esportiva demonstram maior percepção de autoeficácia e experiência positiva no esporte. Holt (2008) corroboram com esses resultados, destacando que quanto mais tempo os jovens se mantêm engajados em uma atividade, maior será o comprometimento e o envolvimento nas atividades, contribuindo para a qualidade das experiências adquiridas.

Foi possível verificar que na relação treinadoratleta, quanto mais comprometimento existir, maior será a proximidade e a complementaridade existente entre ambos. Quanto mais os treinadores e atletas mantiveram-se comprometidos, desenvolver uma amizade positiva, cooperativa e equilibrada, maior a interdependência entre eles (Mata \& Gomes, 2016). A qualidade das relações interpessoais influência no engajamento do atleta na tarefa e comprometimento com o treinador (Cheuczuk et al., 2016; Hampson \& Jowett, 2014; Jowett \& Ntoumanis, 2004). Nos estudos de Contreira et al. (2019) e Davis et al. (2019) foram associados positivamente com a percepção de satisfação dos atletas com o esporte. Quanto mais os atletas estabelecem relacionamentos interpessoais positivos com os seus treinadores, mais satisfeitos sentem-se com o esporte.

Estudo de Cheuczuk et al. (2016) identificou que quanto mais os atletas se percebem próximos e acolhidos pelo treinador, maior foi o foco na tarefa, ressaltando a tendência em vivenciarem um clima motivacional positivo (Adie \& Jowett, 2010; Alfermann, Geisler, \& Okade, 2013). Hampson e Jowett (2014) mostram que quanto melhor a percepção do relacionamento treinador-atleta, maior é a preocupação do atleta com o desenvolvimento das habilidades pessoais e o comprometimento da equipe em alcançar as metas. Jowett e Ntoumanis (2004) destacam a importância do atleta se sentir próximo e 


\section{Relação Coach-athlete e experiência positiva da juventude}

comprometido com seu treinador para que ocorra o desenvolvimento da orientação a metas (Alfermann et al., 2013), eficácia coletiva (Hampson \& Jowett, 2014; Kawazu, Sugiyama, \& Nakasuga, 2012), a coesão de grupo (Jowett \& Chaundy, 2004) e o sucesso esportivo (Jowett \& Cockerill, 2003). Portanto, a combinação de um comportamento de liderança do treinador e a qualidade da relação treinador-atleta fornecem as melhores oportunidades de experiências positivas de desenvolvimento no esporte.

É consenso que as experiências positivas no esporte estão ligadas à qualidade das relações interpessoais que os atletas desenvolvem com os treinadores, pais e colegas (Fraser-Thomas, Côté, \& Deakin, 2005; Hodge, Kanters, Forneris, Bocarro, \& McCord, 2017; Jowett \& Cockerill, 2003). Contudo, a partir do nosso estudo, fica demonstrada a influência de outros fatores para explicar a relação treinadoratleta e experiência positiva dos jovens no esporte. A relação treinador-atleta, considerando o contexto da prática esportiva e tempo de experiência, pode não influenciar a experiência positiva dos jovens no esporte em termos de habilidades e comportamento. Assim, a experiência positiva no esporte é percebida, mas não foi explicada pela qualidade da relação treinador-atleta por si.

Em relação às diferenças observadas por modalidade, é preciso realizar outros estudos. Não encontramos estudos realizados observando diferentes modalidades. Os estudos de Vella et al. (2013), Vieira et al. (2015a) e Fiorese et al. (2018), foram realizados respectivamente nas modalidades de futebol $\mathrm{e}$ voleibol. Em se tratando de diferenças entre modalidades, o perfil dos treinadores e fatores contextuais podem exercer influência, como calendário de competições e as características estruturais e funcionais da modalidade.

$\mathrm{O}$ gênero não se apresentou como um fator que influenciou a qualidade da relação treinadoratleta, nem a percepção da experiência positiva dos jovens no esporte escolar extracurricular. Para a relação treinador-atleta, percebemos que o gênero tem sido pouco explorado nos estudos, sendo apenas considerado no total da amostra. Para a experiência positiva dos jovens no esporte tem sido documentada diferença (Reverdito et al., 2017a), especialmente em relação às habilidades pessoais e sociais (Nascimento Junior et al., 2019). Contudo, estudos com diferentes abordagens teóricas e metodológicas têm associado o gênero à qualidade da relação treinador-atleta como determinante para a experiência positiva no esporte (de Haan \& Norman, 2020). Logo, a qualidade da relação treinador-atleta precisa ser mais bem investigada em se tratando do gênero.

Assim, a partir desses resultados, destacamos a necessidade de conhecer melhor o treinador no contexto de esporte escolar extracurricular. No Brasil, a escola é o principal espaço de acesso à prática esportiva. Porém, há poucos estudos sobre treinadores que atuam no esporte em nível escolar, por exemplo, precisamos entender melhor seu conhecimento profissional, tempo de atuação, condições de trabalho e calendário esportivo. Essas informações são importantes para compreender a experiência dos jovens no esporte e o papel do treinador no desenvolvimento positivo dos jovens.

\section{CONCLUSÃO}

O objetivo deste estudo foi verificar se a qualidade da relação treinador-atleta está relacionada à percepção da experiência positiva dos jovens no esporte. Os resultados encontrados sugerem que a qualidade da relação treinador-atleta não influencia a experiência positiva dos jovens no esporte, no contexto escolar extracurricular. O contexto e o tempo de engajamento na prática esportiva precisam ser considerados na relação treinador-atleta e na experiência positiva dos jovens no esporte. Portanto, é possível concluir que a qualidade da relação treinadoratleta exerce influência sobre a experiência positiva dos jovens no esporte, mas pode não ser o principal preditor no esporte escolar extracurricular.

A modalidade foi associada positiva e significativamente com a qualidade da relação treinador-atleta. O tempo de treino foi associado positiva e significativamente com a percepção de comprometimento treinador-atleta e experiência positiva dos jovens no esporte. Deste modo, quanto mais tempo os atletas estão inseridos em uma prática esportiva, maiores poderão ser as experiências esportivas obtidas e melhores os resultados, favorecendo os benefícios obtidos por meio da relação treinador-atleta.

Embora as evidências deste estudo tenham destacado que a qualidade da relação treinador-atleta explicou parcialmente a percepção da experiência positiva no esporte, algumas limitações precisam ser apontadas. Verificamos apenas jovens atletas 


\section{Tavares, M. A.; Fonseca, S.; Lopes, A.; Galatti, L. R.; Reverdito, R. S.}

participantes do contexto escolar, sendo necessário explorar outros níveis de envolvimento com a prática esportiva. Além disso, pelo fato dos estudos sobre a relação treinador-atleta e experiência positiva dos jovens no esporte estarem concentrados em países desenvolvidos, ocorre a necessidade de estudos em outros contextos, uma vez que fatores socioeconômicos interferem nas condições de prática esportiva. Também, sugerimos que estudos futuros considerem o gênero e as características particulares da organização competitiva (quadro competitivo, planeamento, distância) do contexto investigado.

\section{APLICAÇÕES PRÁTICAS}

Como implicações práticas desta pesquisa, os resultados contribuem para a intervenção dos treinadores, evidenciando o quanto é essencial que se construa um ambiente de treino harmônico, pautado em relações interpessoais positivas com os atletas, de modo que tenham interesse em permanecer no contexto de prática esportiva por um longo período de tempo. Também, constituem diretrizes relevantes para seleção de conteúdos para os programas de formação de treinadores e gestores do esporte, especialmente em nível escolar. Quanto mais treinadores e atletas desenvolverem relações interpessoais baseadas no respeito, confiança, afeto, cooperação, ligação e apresentarem interesse em manter a qualidade desta relação, maior a interdependência entre eles e, consequentemente, melhor poderá ser a experiência dos jovens no esporte.

\section{AGRADECIMENTOS}

As autoras e autores agradecem: colaboração dos profissionais da Secretaria Adjunta de Esporte e Lazer do estado de Mato Grosso (SAEL) e os gestores e professores/treinadores, responsáveis pela realização dos Jogos Escolares da Juventude do estado de Mato Grosso; apoio dos pesquisadores e alunos do Centro Interdisciplinar de Pesquisas em Esporte e Exercício Físico (CIPEEF).

\section{CONFLITOS DE INTERESSE}

Nada a declarar.

\section{FINANCIAMENTO}

As autores e autores divulgam que este trabalho foi financiado: Fundação de Amparo à Pesquisa do Estado de Mato Grosso (Processo: FAPEMAT.0590374/2016); Programa REDE CEDES do Estado de Mato Grosso, Ministério da Cidadania/Secretaria Especial do Esporte (Convênio: 824066/2015). Tavares, M.A., foi apoiada com uma bolsa de estudos da Coordenação de Aperfeiçoamento de Pessoal de Nível Superior (CAPES).

\section{REFERÊNCIAS}

1. Adie, J. W., \& Jowett, S. (2010). MetaPerceptions of the Coach-Athlete Relationship, Achievement Goals, and Intrinsic Motivation Among Sport Participants. Journal of Applied Social Psychology, 40(11), 2750-2773. https://doi.org/10.1111/j.15591816.2010.00679.x

2. Alfermann, D., Geisler, G., \& Okade, Y. (2013). Goal orientation, evaluative fear, and perceived coach behavior among competitive youth swimmers in Germany and Japan. Psychology of Sport and Exercise, 14(3), 307-315. https://doi.org/10.1016/j.psychsport.2012.11.005

3. Aoyagi, K., Ishii, K., Shibata, A., Arai, H., Fukamachi, H., \& Oka, K. (2020). A qualitative investigation of the factors perceived to influence student motivation for school-based extracurricular sports participation in Japan. International Journal of Adolescence and Youth, 25(1), 624-637. https://doi.org/10.1080/02673843.2019.1700139

4. Brenda Light, B., \& David Light, S. (2006). Sports and Character Development. Journal of Physical Activity and Health, 3(2), 255-256. https://doi.org/10.1123/jpah.3.2.255

5. Camiré, M., Rathwell, S., Turgeon, S., \& Kendellen, K. (2019). Coach-athlete relationships, basic psychological needs satisfaction and thwarting, and the teaching of life skills in Canadian high school sport. International Journal of Sports Science \& Coaching, $\quad$ 14(5), 591-606. https://doi.org/10.1177/1747954119869542

6. Camiré, M., \& Trudel, P. (2010). High school athletes' perspectives on character development through sport participation. Physical Education and Sport Pedagogy, 15(2), 193-207. https://doi.org/10.1080/17408980902877617 


\section{Relação Coach-athlete e experiência positiva da juventude}

7. Cheuczuk, F., Ferreira, L., Flores, P. P., Vieira, L. F., Vieira, J. L. L., \& Nascimento Junior, J. R. A. d. (2016). Qualidade do Relacionamento Treinador-Atleta e Orientação às Metas como Preditores de Desempenho Esportivo. Psicologia: Teoria e Pesquisa, 32.

8. Coatsworth, J. D., \& Conroy, D. E. (2009). The effects of autonomy-supportive coaching, need satisfaction, and self-perceptions on initiative and identity in youth swimmers. Developmental Psychology, 45(2), 320-328. https://doi.org/10.1037/a0014027

9. Contreira, A. R., Nascimento Junior, J. R. A. d., Caruzzo, N. M., Costa, L. C. A. d., Gaion, P. A., Melo, S. V. A., \& Fiorese, L. (2019). Basic Psychological Needs and Sports Satisfaction Among Brazilian Athletes and Coaches: The Mediating Role of the Dyadic Relationship. Frontiers in Psychology, 10, 2543. https://doi.org/10.3389/fpsyg.2019.02543

10. Côté, J., \& Hancock, D. J. (2016). Evidencebased policies for youth sport programmes. International Journal of Sport Policy and Politics, $\quad 8(1), \quad$ 51-65. https://doi.org/10.1080/19406940.2014.919338

11. Côté, J., Strachan, L., \& Fraser-Thomas, J. (2008). Participation, personal development, and performance through youth sport. In N. L. Holt (Ed.), Positive Youth Development Through Sport (pp. 34-45). London: Routledge.

12. Coutinho, P., Mesquita, I., \& Fonseca, A. M. (2018). Parental influences in athlete sport participation: a systematic review of literature. Revista de Psicología del Deporte, 27(2), 47-58.

13. Cronin, L. D., \& Allen, J. B. (2015). Developmental experiences and well-being in sport: The importance of the coaching climate. The Sport Psychologist, 29(1), 62-71. https://doi.org/10.1123/tsp.2014-0045

14. Danish, S., Forneris, T., Hodge, K., \& Heke, I. (2004). Enhancing Youth Development Through Sport. World Leisure Journal, 46(3), 38-49. https://doi.org/10.1080/04419057.2004.9674365

15. Davis, L., Jowett, S., \& Tafvelin, S. (2019). Communication Strategies: The Fuel for Quality Coach-Athlete Relationships and Athlete Satisfaction. Frontiers in Psychology, 10, 2156. https://doi.org/10.3389/fpsyg.2019.02156

16. de Haan, D., \& Norman, L. (2020). Mind the gap: the presence of capital and power in the female athlete-male -coach relationship within elite rowing. Sports Coaching Review, 9(1), 95118.

https://doi.org/10.1080/21640629.2019.1567160

17. Fiorese, L., Pizzo, G. C., Contreira, A. R., LazierLeão, T. R., Moreira, C. R., Rigoni, P. A. G., \& do Nascimento Junior, J. R. A. (2018). Association between motivation and group cohesion in professional football: Is the coachathlete relationship a determining factor? Revista de Psicología del Deporte, 27(4, Supp, 1), 51-57.

18. Fraser-Thomas, J. L., Côté, J., \& Deakin, J. (2005). Youth sport programs: an avenue to foster positive youth development. Physical Education and Sport Pedagogy, 10(1), 19-40. https://doi.org/10.1080/1740898042000334890

19. Gaion, P., Milistetd, M., Santos, F., Contreira, A., Arantes, L., \& Caruzzo, N. (2020). Coaching Positive Youth Development in Brazil: Recommendations for Coach Education Programs. International Sport Coaching Journal, 7(1), 82-88. https://doi.org/10.1123/iscj.2018$\underline{0106}$

20. Galatti, L. R., Côté, J., Reverdito, R. S., Allan, V., Seoane, A. M., \& Paes, R. R. (2017). Fostering Elite Athlete Development and Recreational Sport Participation: a Successful Club Environment. Motricidade; Vol 12, No 3 (2016). https://doi.org/10.6063/motricidade.6099

21. Gilman, R. (2001). The relationship between life satisfaction, social interest, and frequency of extracurricular activities among adolescent students. Journal of Youth and Adolescence, 30(6), 749-767. https://doi.org/10.1023/a:1012285729701

22. Gould, D., \& Carson, S. (2008). Life skills development through sport: current status and future directions. International Review of Sport and Exercise Psychology, 1(1), 58-78. https://doi.org/10.1080/17509840701834573

23. Gould, D., \& Carson, S. (2010). The relationship between perceived coaching behaviors and developmental benefits of high school sports participation. Hellenic Journal Psychology, 7(3), 298-314.

24. Gould, D., Flett, R., \& Lauer, L. (2012). The relationship between psychosocial developmental and the sports climate experienced by underserved youth. Psychology of 


\section{Tavares, M. A.; Fonseca, S.; Lopes, A.; Galatti, L. R.; Reverdito, R. S.}

Sport and Exercise, 13(1), 80-87. https://doi.org/10.1016/j.psychsport.2011.07.005

25. Grubertt, G. A., Cavazzotto, T., Junior, A. V., Mouad, M., Dib, L. R. P., Junior, H. S., \& Dourado, A. C. (2020). Motives of participation in Paraná School Games of students-athlets in Brazil. Cuadernos de Psicología del Deporte, 20(2). https://doi.org/10.6018/cpd.347661

26. Hampson, R., \& Jowett, S. (2014). Effects of coach leadership and coach-athlete relationship on collective efficacy. Scandinavian Journal of Medicine \& Science in Sports, 24(2), 454-460. https://doi.org/10.1111/j.16000838.2012.01527.x

27. Hansen, D. M., \& Larson, R. W. (2007). Amplifiers of developmental and negative experiences in organized activities: Dosage, motivation, lead roles, and adult-youth ratios. Journal of Applied Developmental Psychology, 28(4), 360-374. https://doi.org/10.1016/j.appdev.2007.04.006

28. Hansen, D. M., Larson, R. W., \& Dworkin, J. B. (2003). What Adolescents Learn in Organized Youth Activities: A Survey of Self-Reported Developmental Experiences. Journal of Research on Adolescence, 13(1), 25-55.

29. Hodge, C. J., Kanters, M. A., Forneris, T., Bocarro, J. N., \& -McCord, R. S. (2017). A Family Thing: Positive Youth Development Outcomes of a Sport-Based Life Skills Program. Journal of Park and Recreation Administration, 35(1), 34-50. https://doi.org/10.18666/Jpra2017-V35-I1-6840

30. Holt, N. L. (2008). Positive youth development through sport. New York: Routledge.

31. Holt, N. L., Neely, K. C., Slater, L. G., Camire, M., Cote, J., Fraser-Thomas, J., . . . Tamminen, K. A. (2017). A grounded theory of positive youth development through sport based on results from a qualitative meta-study. Int Rev Sport Exerc Psychol, 10(1), 1-49. https://doi.org/10.1080/1750984X.2016.118070 $\underline{4}$

32. Jowett, S. (2005). The coach-athlete partnership. The Psychologist, 18(7), 412-415.

33. Jowett, S. (2009). Validating Coach-Athlete Relationship Measures with the Nomological Network. Measurement in Physical Education and Exercise Science, 13(1), 34-51. https://doi.org/10.1080/10913670802609136
34. Jowett, S., \& Chaundy, V. (2004). An Investigation Into the Impact of Coach Leadership and Coach-Athlete Relationship on Group Cohesion. Group Dynamics: Theory, Research, and Practice, 8(4), 302-311. https://doi.org/10.1037/1089-2699.8.4.302

35. Jowett, S., \& Cockerill, I. M. (2003). Olympic medallists' perspective of the althlete-coach relationship. Psychology of Sport and Exercise, $4(4)$, 313-331. https://doi.org/10.1016/S14690292(02)00011-0

36. Jowett, S., \& Ntoumanis, N. (2004). The CoachAthlete Relationship Questionnaire (CART-Q): development and initial validation. Scandinavian Journal of Medicine \& Science in Sports, 14(4), 245-257. $\quad$ https://doi.org/10.1111/j.16000838.2003.00338.x

37. Jowett, S., \& Poczwardowski, A. (2007). Understanding the Coach-Athlete Relationship. Social Psychology in Sport. (pp. 3-14). Champaign, IL, US: Human Kinetics.

38. Kawazu, K., Sugiyama, Y., \& Nakasuga, T. (2012). An Investigation of the Changing Relationship between the Collective Efficacy and Team Performance of a Sport Team for Different Sporting Events. Japanese Journal of Sport Psychology, 39(2), 153-167. https://doi.org/10.4146/jispopsy.2012-1120

39. Larson, R. W. (2000). Toward a psychology of positive youth development. American Psychologist, 55(1), 170-183. https://doi.org/10.1037/0003-066X.55.1.170

40. Larson, R. W., Hansen, D. M., \& Moneta, G. (2006). Differing profiles of developmental experiences across types of organized youth activities. Dev Psychol, 42(5), 849-863. https://doi.org/10.1037/0012-1649.42.5.849

41. Larson, R. W., Perry, S. C., Hang, H., \& Walker, K. C. (2011). New Horizons: Understanding the Processes and Practices of Youth Development. Journal of Youth Development: Bridging Research \& Practice, 6(3), 155-156.

42. Larson, R. W., \& Tran, S. P. (2014). Invited commentary: Positive youth development and human complexity. J Youth Adolesc, 43(6), 10121017. https://doi.org/10.1007/s10964-014-0124$\underline{9}$

43. Lima, L. A. d. (2018). Carreira esportiva: um estudo com atletas de excelência. Mestra em Educação Física, Universidade Estadual de 


\section{Relação Coach-athlete e experiência positiva da juventude}

Campinas, Campinas. Retrieved from http://repositorio.unicamp.br/bitstream/REPOSI P/335431/1/Lima_LeilaneAlvesDe_M.pdf

44. MacDonald, D., Côté, J., \& Deakin, J. (2010). The Impact of Informal Coach Training on the Personal Development of Youth Sport Athletes. International Journal of Sports Science and Coaching, 5(3), 363-372.

45. MacDonald, D. J., Cote, J., Eys, M., \& Deakin, J. (2012). Psychometric properties of the youth experience survey with young athletes. Psychology of Sport and Exercise, 13(3), 332340.

https://doi.org/10.1016/j.psychsport.2011.09.001

46. Mageau, G. A., \& Vallerand, R. J. (2003). The coach-athlete relationship: a motivational model. Journal of Sports Sciences, 21(11), 883-904. https://doi.org/10.1080/0264041031000140374

47. Martín de Benito, M., José Francisco, G. L., \& BenitoTrigueros, A. M. d. (2018). Inteligencia emocional, percepción de apoyo a la autonomía y relaciones en el deporte. Cuadernos de Psicología del Deporte, 18(1).

48. Mata, R. T., \& Gomes, A. R. (2016). Relação treinador-atleta: Teoria, investigação e intervenção. In D. Bartholomeu, J. M. Montiel, A. A. Machado, A. R. Gomes, G. Couto \& V. Cassepp-Borges (Eds.), Relações interpessoais: Concepções e Contextos de Intervenção $e$ Avaliação (pp. 155-174). São Paulo: Vetor Editora.

49. Mollerlokken, N. E., Loras, H., \& Pedersen, A. V. (2017). A Comparison of Players' and Coaches' Perceptions of the Coach-Created Motivational Climate within Youth Soccer Teams. Frontiers in Psychology, 8. https://doi.org/10.3389/fpsyg.2017.00109

50. Nascimento Junior, J. R. A. d., da Silva, A. A., Granja, C. T. L., Oliveira, D. V. d., Reis Batista, R. P., \& Fortes, L. d. S. (2019). ¿Las experiencias deportivas predicen la cohesión del equipo en atletas jóvenes? Cuadernos de Psicología del Deporte, 19(3). https://doi.org/10.6018/cpd.365201

51. Naylor, A. H. (2006). The Coach's Dilemma: Balancing Playing to Win and Player Development. The Journal of Education, 187(1), 31-48.

52. Nicholls, A. R., Earle, K., Earle, F., \& Madigan, D. J. (2017). Perceptions of the Coach-Athlete
Relationship Predict the Attainment of Mastery Achievement Goals Six Months Later: A TwoWave Longitudinal Study among F. A. Premier League Academy Soccer Players. Frontiers in Psychology, $8, \quad 684$. https://doi.org/10.3389/fpsyg.2017.00684

53. Nicholls, A. R., \& Perry, J. L. (2016). Perceptions of Coach-Athlete Relationship Are More Important to Coaches than Athletes in Predicting Dyadic Coping and Stress Appraisals: An ActorPartner Independence Mediation Model. Frontiers in Psychology, 7, 447. https://doi.org/10.3389/fpsyg.2016.00447

54. Orozco, A. M., Perez, A. A., Sierra, F. S., Supervia, P. U., \& Jarie, L. (2018). Emerging learning outcomes in Under-7 football competition: towards the adherence, droppingout or aggressiveness. Sportis-Scientific Technical Journal of School Sport Physical Education and Psychomotricity, 4(1), 184-205. https://doi.org/10.17979/sportis.2018.4.1.2151

55. Papacharisis, V., Goudas, M., Danish, S. J., \& Theodorakis, Y. (2005). The Effectiveness of Teaching a Life Skills Program in a Sport Context. Journal of Applied Sport Psychology, 17(3), 247-254. https://doi.org/10.1080/10413200591010139

56. Reece, L. J., McInerney, C., Blazek, K., Foley, B. C., Schmutz, L., Bellew, B., \& Bauman, A. E. (2020). Reducing financial barriers through the implementation of voucher incentives to promote children's participation in community sport in Australia. BMC Public Health, 20(1). https://doi.org/10.1186/s12889-019-8049-6

57. Reverdito, R. S., Carvalho, H. M., Galatti, L. R., Scaglia, A. J., Goncalves, C. E., \& Paes, R. R. (2017a). Effects of Youth Participation in ExtraCurricular Sport Programs on Perceived SelfEfficacy: A Multilevel Analysis. Perceptual and Motor Skills, 124(3), 569-583. https://doi.org/10.1177/0031512517697069

58. Reverdito, R. S., Galatti, L. R., Carvalho, H. M., Scaglia, A. J., Cote, J., Goncalves, C. E., \& Paes, R. R. (2017b). Developmental Benefits of Extracurricular Sports Participation Among Brazilian Youth. Perceptual and Motor Skills, 124(5), 946-960. https://doi.org/10.1177/0031512517724657

59. Reverdito, R. S., Galatti, L. R., Lima, L. A. d., Nicolau, P. S., Scaglia, A. J., \& Paes, R. R. 


\section{Tavares, M. A.; Fonseca, S.; Lopes, A.; Galatti, L. R.; Reverdito, R. S.}

(2016). The "Programa Segundo Tempo" in Brazilian municipalities: outcome indicators in macrosystem. Journal of Physical Education, 27(e2754), $1-14$. https://doi.org/10.4025/jphyseduc.v27i1.2754

60. Reverdito, R. S., Galatti, L. R., Scaglia, A. J., \& Paes, R. R. (2020a). Youth Participation in Sport in a Context of Vulnerability: Perception of Positive and Negative Experiences. EBalonmano Com, 16(1), 55-66.

61. Reverdito, R. S., Galatti, L. R., Strachan, L., Scaglia, A. J., \& Paes, R. R. (2020b). Coaching and continuity make a difference: competence effects in a youth sport program. Journal of Physical Education and Sport, 20(4), 1964-1971. https://doi.org/10.7752/jpes.2020.04266

62. Rigoni, P. A. G. (2014). Contribuição $d a$ Experiência esportiva positiva, mediada Pela Motivação, não Propósito de Vida de Jovens Atletas brasileiros. Doutora, Universidade Estadual de Maringá (UEM), Maringá-PR.

63. Rodrigues, A. I. C., Evans, M. B., \& Galatti, L. R. (2019). Social identity and personal connections on the mat: Social network analysis within Brazilian Jiu-Jitsu. Psychology of Sport and Exercise, 40, 127-134. doi: https://doi.org/10.1016/j.psychsport.2018.10.006

64. Santos, F., Gould, D., \& Strachan, L. (2019). Research on Positive Youth Developmentfocused Coach Education Programs: Future Pathways and Applications. International Sport Coaching Journal, 6(1), 132-138. https://doi.org/10.1123/iscj.2018-0013

65. Santos, F., \& Martinek, T. J. (2018). Facilitating Positive Youth Development through Competitive Youth Sport: Opportunities and Strategies. Strategies, 31(6), 47-49. https://doi.org/10.1080/08924562.2018.1516443

66. Santos, F. d. S. F. d., Camiré, M., \& Campos, P. H. d. F. (2018). Youth sport coaches' role in facilitating positive youth development in Portuguese field hockey. International Journal of Sport and Exercise Psychology, 16(3), 221-234. https://doi.org/10.1080/1612197x.2016.1187655

67. Sheridan, D., Coffee, P., \& Lavallee, D. (2014). A systematic review of social support in youth sport. International Review of Sport and Exercise Psychology, 7(1), 198-228. https://doi.org/10.1080/1750984x.2014.931999
68. Smith, A. L., Ullrich-French, S., Walker, E., \& Hurley, K. S. (2006). Peer Relationship Profiles and Motivation in Youth Sport. Journal of Sport and Exercise Psychology, 28(3), 362-382. https://doi.org/10.1123/jsep.28.3.362

69. Turnnidge, J., Côté, J., \& Hancock, D. J. (2014). Positive Youth Development From Sport to Life: Explicit or Implicit Transfer? Quest, 66(2), 203217. doi: https://doi.org/10.1080/00336297.2013.867275

70. Vella, S., Oades, L., \& Crowe, T. (2011). The Role of the Coach in Facilitating Positive Youth Development: Moving from Theory to Practice. Journal of Applied Sport Psychology, 23(1), 3348. doi: https://doi.org/10.1080/10413200.2010.511423

71. Vella, S. A., Oades, L. G., \& Crowe, T. P. (2013). The relationship between coach leadership, the coach-athlete relationship, team success, and the positive developmental experiences of adolescent soccer players. Physical Education and Sport Pedagogy, 18(5), 549-561. https://doi.org/10.1080/17408989.2012.726976

72. Vieira, J. L. L., Ferreira, L., Cheuczuk, F., Flores, P. P., Vissoci, J. R. N., Rocha, F. F. d., . . Vieira, L. F. (2015a). Impact of coach-athlete relationship on the collective efficacy of young volleyball players. Brazilian Journal of Kinanthropometry and Human Performance; Vol 17, No 6 (2015). https://doi.org/10.5007/1980$0037.2015 \mathrm{v} 17 \mathrm{n} 6 \mathrm{p} 650$

73. Vieira, L. F., Nascimento Junior, J. R. A. d., Pujals, C., Jowett, S., Codonhato, R., \& Vissoci, J. R. N. (2015b). Cross-cultural adaptation and psychometric properties of the Brazilian coachathlete relationship questionnaire (CART-Q) Athlete Version. Revista Brasileira de Cineantropometria \& Desempenho Humano, 17, 635-649. $\quad$ https://doi.org/10.5007/1980$\underline{0037.2015 \mathrm{v} 17 \mathrm{n} 6 \mathrm{p} 635}$

74. Wachsmuth, S., Jowett, S., \& Harwood, C. G. (2017). Conflict among athletes and their coaches: what is the theory and research so far? International Review of Sport and Exercise Psychology, 10(1), 84-107. https://doi.org/10.1080/1750984x.2016.1184698

75. Wright, A., \& Cote, J. (2003). A retrospective analysis of leadership development through sport. Sport Psychologist, 17(3), 268-291. https://doi.org/10.1123/Tsp.17.3.268 


\section{Relação Coach-athlete e experiência positiva da juventude}

76. Yang, S. X., \& Jowett, S. (2012). Psychometric properties of the Coach-Athlete Relationship Questionnaire (CART-Q) in seven countries.
Psychology of Sport and Exercise, 13(1), 36-43. https://doi.org/10.1016/j.psychsport.2011.07.010 